\section{Reduced suicide risk for affectively ill patients}

Sir - In the September issue of the Journal two papers provided valuable information about suicides and their prevention, ${ }^{1,2}$ but only one of them mentioned lithium and only briefly. There is, however, reason to elaborate on this treatment, which in affectively ill patients at high risk of committing suicide can reduce this risk markedly. Two recent reviews analysed the evidence, from many studies involving large numbers of patients.

In one of them ${ }^{3}$ the average number of suicidal acts was 0.255 per patient-year in manic-depressive patients given lithium and 1.778 per patient-year in such patients not given lithium, a seven-fold difference. The frequency of suicide attempts was 23 times lower and the frequency of completed suicides five time lower in patients on lithium than in those not on lithium.

The other review ${ }^{4}$ dealt exclusively with studies in which the same patients had been followed before, during, and after lithium treatment.

While manic-depressive patients have a mortality that is about three times higher than in the population at large, primarily caused by suicides, the mortality in lithium treated patients was in most studies not significantly higher than in the general population.

This was so for bipolar, unipolar, and schizoaffective patients. In some studies the frequency of suicidal acts was high also during lithium treatment, but it was still higher when lithium was not given. The frequency of suicide attempts was five to 16 times lower and the frequency of completed suicides three to 17 times lower in patients on lithium than in patients not on lithium.

There was accordingly a marked association between prophylactic lithium treatment, on the one hand, and mortality and suicidal behaviour, on the other.

But correlation is not the same as causation, and other factors than lithium may have played a role. The fall in frequency of suicidal acts after start of lithium treatment could for example have been caused by extra care and supervision during the treatment.

That is, however, not likely. Patients for whom long-term lithium treatment was prescribed had had several severe depressions in the past, but repeated hospital stays with their extra care and supervision had no prevented suicide attempts.

The rise in frequency of suicidal acts after discontinuation of lithium could have been caused by a treatment-independent difference between non-compliant and compliant patients. Among the former there is more alcoholism and drug misuse than among the latter, conditions that in themselves carry extra risk of suicide.

This may explain some of the rise, but hardly the whole. In some patients the physician stopped lithium owing to side effect or pregnancy, and it is not likely that such patients had increased frequency of misuse. ${ }^{2}$

It should further be noted that there have been no reports of reduced suicidal behaviour in patients given maintenance treatment with anticonvulsants or anti depressants. During long-term administration of these drugs the same 'other factors' must have been at work as during treatment with lithium, and yet it is only with the latter drug that there is a temporal coincidence of treatment and lowered suicidal behaviour.
It would be wrong to claim that an anti-suicidal action of prophylactic lithium treatment has been definitively proven. Such proof is in fact not possible for any kind of anti-suicidal intervention. One cannot maintain suicide threatened patients on placebo or withhold psychological and social support from them in order to observe when they will kill themselves. But the extensive evidence has not disproved that lithium has an anti-suicidal action. As scientists we must concede that such an action remains a possibility. As responsible physicians we cannot make therapeutic decisions without taking that possibility into consideration.

Lithium should be used as a suicide-preventive drug in carefully diagnosed and conscientiously monitored bipolar, unipolar, and schizo-affective patients with severe depressions or depressions with suicidal ideation or with suicide attempts in the past.

\section{Mogens Schou \\ MD, Dr med Sci, Dr h c, FRCPsych (Hon) Emeritus professor Syrenvej 4 \\ DK-8240 Risskov \\ Denmark}

References

1. Kelleher MJ, Keohane B, Corcoran P, Keeley HS, Neilson S. An investigation of one hundred suicides. Ir J Psych Med 2000; 17(3): 86-90.

2. O'Shea B, Stokes M, Falvey J. Completed suicide: an update for the general psychiatrists. Ir J Psych Med 2000; 17(3): 100-105.

3. Tondo L, Baldessarini RJ. Reduced suicide risk during lithium maintenance treatment. I Clin Psychiatry 2000; 61(suppl 9): 97-1 04.

4. Schou $M$. The effect of prophylactic lithium treatment on mortality and suicidal behaviour: A review for clinicians. J Affect Disord 1998; 50: 253-259.

\section{EBM - a rigorous but common sense approach}

Sir - A recent editorial in this Journal sought to outline a number of issues concerning evidence based medicine (EBM), highlighting several potential advantages and limitations of this approach.

Attention was drawn to two review articles published in the British Journal of Psychiatry, both seeking to appraise the efficacy of lithium. ${ }^{2,3}$ The two authors had come to entirely opposing conclusions and closer examination revealed that each had cited largely different papers during the process of their reviews. It is unfortunate that this was interpreted as demonstrating shortcomings in EBM as neither review was systematic. Their publication under the banner of EBM was subsequently challenged as misleading, ${ }^{4}$ and they might better be described as examples of "prejudice-based medicine", with each author choosing to focus on evidence supporting their pre-existing viewpoint.

An EBM approach would seek to replace this with systematic review, where a method for the review is made explicit, including search and inclusion criteria, and aiming to thereby objectively explore the subject.

The editorial also appeared to propagate a popular misconception that EBM offers a solution to existing problems in research by means solely of meta analysis and systematic review. The underlying principle of EBM is that the best available evidence should be applied to clinical practice. 
This 'best evidence' takes different forms depending upon the nature of the clinical question. For example, questions concerning prognosis, or aetiology would require entirely different strategies, and some questions may best be answered using rigorous qualitative methods, a fact recognised by the journal Evidence Based Mental Health which includes a section for this type of research.

The EBM approach should be seen as a broad, common sense but rigorous method of applying research to every day clinical practice, although with the result often yielding more questions than answers.
Andrew Owen

Specialist Registrar in Psychiatry,

Lyndon Clinic, Hobs Meadow, Solibull, B92 8PW

England

References

1. Casey P. Evidence-based psychiatry: 'Beguiled by certainty'. Ir J Psych Med $2000 ; 17(3): 80-1$

2. Cookson J. Lithium: balancing risks and benefits. Br J Psych 1997; 171: 120-4 3. Moncrieff J. Lithium: evidence reconsidered. Br J Psych 1997; 171:1 13-9

4. Powell JA, Geddes JK. Evidence-based psychiatry. Br J Psych 1997; 171:586-7.

5. Sackett DL, Rosenberg WC, Gray JAM, Haynes RB, Richardson WS

Evidence-based medicine: what it is and what it isn't. BMJ 1996; 312: 71-2.

\section{The John Dunne Award}

The John Dunne Medal was

established to symbolise

excellence and originality in

the field of psychiatric research.

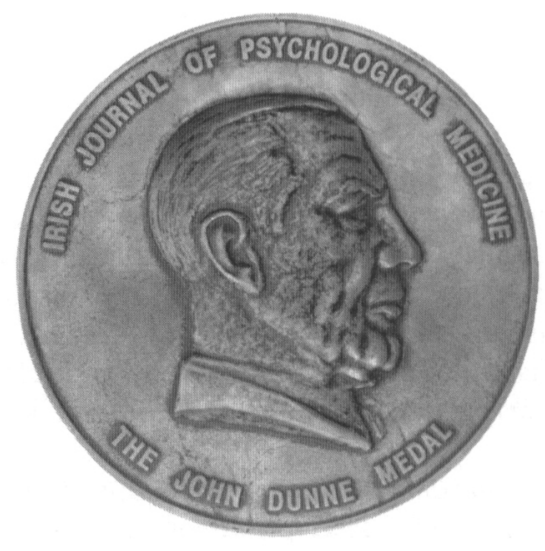

Awarded annually, this prestigious prize from the

Irish Journal of Psychological Medicine

is open to all trainees from Ireland, Northern Ireland and Britain.

To be eligible, a trainee must have made a

significant contribution

(though not necessarily as the first author) to an original paper

published by the Journal over the year.

We welcome submissions in 2001 from trainees who have made a contribution to the field of psychiatric research

through original papers.

The winner will be judged by an international panel. 